\title{
Do Warmups Predict Pole Vault Competition Performance?
}

\author{
Alex Peskin \\ Honors College, Grand V alley State University, Allendale, MI \\ https:/ / doi.org/10.33697/ ajur.2021.044 \\ Student:peskinal@mail.gvsu.edu* \\ Mentor:deanern@gvsu.edu
}

\begin{abstract}
The aim of this research was to determine the relationship between pole vault warmup and competition performance in a sample of 16 collegiate vaulters over 60 observations. Pole vault athletes are given time to warm up in the same area that the competition will take place. This prompted investigation into whether better warmup performance could indicate better familiarity with the performance environment, and whether this could translate to the competition. The number of warmup vaults taken was also considered. Participants were observed during multiple warmup periods and data was collected on warmup performance. The findings indicate a significant correlation between instances in which participants displayed their best warmup scores and their best competition performances, likewise with their worst. Also, participants who took more warmup vaults performed significantly better on average. Athletes and coaches should consider implementing warmup practices that emphasize familiarizing oneself with their performance environment.
\end{abstract}

\section{KEYWORDS}

Pole Vault; Track and Field; Warmups; Warmup Performance; Competition Performance; Performance Environment; Nested Task; Task Constraints

\section{INTRODUCTION}

Compared to practice, competitions often require athletes to perform under new or more challenging circumstances. The competition conditions external to the athlete constitute the performance environment, and can influence multiple skills relevant to athletic performance such as perceiving distance, ${ }^{1,2}$ memorizing distance, ${ }^{3}$ and perceiving velocity. ${ }^{4}$ Familiarity enhances one's ability to perform physical tasks such as navigating, ${ }^{5}$ coordinating motor responses, ${ }^{6}$ and approaching a predetermined target within that environment. ${ }^{7}$ This relationship is also evident when considering the phenomena of the "home field advantage" with home teams winning significantly more competitions across multiple sports and leagues. ${ }^{8}$ In soccer, this effect was significantly reduced when home teams changed stadiums, suggesting the advantage came from familiarity with the conditions of the arena. ${ }^{9}$ This raises the question whether increasing one's familiarity with their performance environment would positively influence their performance within a competition.

Track and field events such as the long jump and pole vault are unique to other events and sports in that athletes are allowed a "warmup period" before the event starts using the same area and performing similar jumps to what they will during the competition. In the case of the pole vault, the warmup period is usually 30-60 minutes long and athletes will take turns using the runway and landing area to perform multiple vaults as they will eventually in the competition. Panteli et al. (2016) explored how the environment and task influenced long jumpers' visual step regulation and found that jumpers exhibited better step regulation and accuracy when the task more closely mimicked a full long jump sequence rather than shortened drills. ${ }^{7}$ They also exhibited better performance on approach drills when performing this task on the long jump runway they normally used rather than a flat track. These results indicate that familiarity with the environment and executing the complete task leads to better performance of elements within that task. Completion of a vault in the pole vault occurs when the athlete builds speed on the runway, plants their pole into a box, swings upside down, and finally launches themselves into the air in an attempt to clear a horizontal bar. Every one of these distinct stages is vital to a successful pole vault attempt, ${ }^{10}$ making the pole vault an ideal event to investigate the importance of task completion.

The current study investigated the relationship between warmup and competition performance in the pole vault. Besides there being no bar to clear during the warmup period, the warmup and competition environments are held constant, making a measure of the completeness of each vault an ideal indicator for warmup performance. Based on the prior research, a higher level of completeness of warmup vaults will indicate better mastery of the competition environment. The total number of warmup vaults will also be considered as repetition contributes to familiarity.11,12 We hypothesize that better performance during the warmup period, as measured by vault completeness, will be associated with better performance during the competition. Based on the research pertaining to familiarity and performance cited earlier, ${ }^{5-7,11,12}$ we also hypothesize that more warm up vaults will be 
associated with better competition performance. The findings of this study may provide insight into the relationship between performance and environment within the pole vault and may help coaches increase the effectiveness of practices during the warmup period.

\section{METHODS}

\section{Participants}

A total of 16 pole vaulters, 7 male and 9 female, participated in the current study and were selected based on convenience. The number of males in their first, second, third, and fourth year of collegiate competition were 4, 0,2 , and 1 , respectively ( $\mathrm{M}=2.00$, $\mathrm{SD}=1.20)$. The number of females were $1,2,5$, and 1 , respectively $(\mathrm{M}=2.67, \mathrm{SD}=.82)$. All participants were NCAA Division II collegiate athletes attending a midwestern university and ranged from 18-21 years of age at the start of the study. The average personal records, in meters, for males and females at the start of the study were $4.58 \mathrm{~m}(\mathrm{SD}=.33)$ and $3.62 \mathrm{~m}(\mathrm{SD}=.33)$, respectively. This sample demographic was chosen because competition at this level requires a substantial degree of technical proficiency that allowed standardization of measures that were based on form. Written informed consent was obtained from all of the participants. Approval for this study was obtained from the university's institutional review board (reference number: 20-117H) and athletic department.

\section{Procedure}

Testing occurred at the same indoor track and field arena at seven different pole vault competitions that adhered to NCAA rules and regulations. These took place over the course of 45 days. The participants were observed by one of two trained research assistants during the warmup period only, both who had extensive prior knowledge and experience with the pole vault. A test of interrater reliability was conducted where both observers independently scored the same 16 warmup vaults performed by four different participants (four vaults each) during the first pole vault competition of the study. The scores were then compared via Pearson correlation, $r(14)=.97, p<.001$. Observers were instructed to interact with the participants as little as possible. Data was collected on each warmup jump performed by each participant. Data was only collected on warmup jumps from the longest approach used by the participants in warmups in an effort to match the data with expected competition conditions. The data was written on a coded template after each vault.

The data recorded during observations consisted of the completeness score of each warmup vault for each participant that was competing that day (Table 1). Completeness was measured on a 0-3 scale based on how far the participant proceeded through the stages of the vault: a score of zero represents a vault in which the participant did not leave the ground, a score of one represents when the participant left the ground but made little or no movement to swing the body upside down, a score of two represents when the participant left the ground and swung upside down so their legs went past parallel with the ground, and a score of three represents when the participant left the ground, swung upside down, and made a concerted effort to mimic clearing a bar as they would in a competition (Figure 1). An obstacle such as a practice bungee did not need to be present to achieve a score of three.

\begin{tabular}{|c|c|c|c|c|c|c|c|}
\hline \multirow{2}{*}{ Participant } & \multicolumn{7}{|c|}{ Warmup Vault Number } \\
\cline { 2 - 9 } & 1 & 2 & 3 & 4 & 5 & 6 & 7 \\
\cline { 2 - 9 } & \multicolumn{7}{|c|}{ Completeness Score on 0-3 Scale } \\
\hline A & 3 & 2 & 1 & 3 & 2 & 1 & NA \\
\hline B & 0 & 0 & 1 & 1 & 2 & 3 & NA \\
\hline C & 1 & 1 & 2 & 2 & 3 & NA \\
\hline
\end{tabular}

Table 1. Example of fictional observational data. "NA" is used in instances where the participant took less than that number of warmups.

Apart from the data collected during observations, the participants' performance in the competitions as well as their personal records were obtained from the public domain via "athletic.net."

Participants' competition performance was measured by using a ratio of the height scored in competition $(\mathrm{CH})$ to the participant's personal record (PR) at the time of that competition. This allowed for the comparison of all the participants regardless of individual differences in competency or experience. This calculation resulted in a performance score typically ranging from zero (in the case a participant did not make any height) to one (the participant tied their PR). If a participant vaulted a new PR, a score greater than one would result, and the new PR would be used in the calculation for subsequent competitions. This was done to control for any maturation that occurred over the course of the study.

The main analyses consisted of comparing competition performance with multiple variations of the warmup scores via Pearson correlation. Competition performance was also compared with the total number of warmup vaults taken (TWUV). In addition to 
the average scores for all of the warmup vaults for a given competition (S), the average of all of the warmup vaults except the first (SE1) and the average of the last three warmup vaults (SL3) were included.

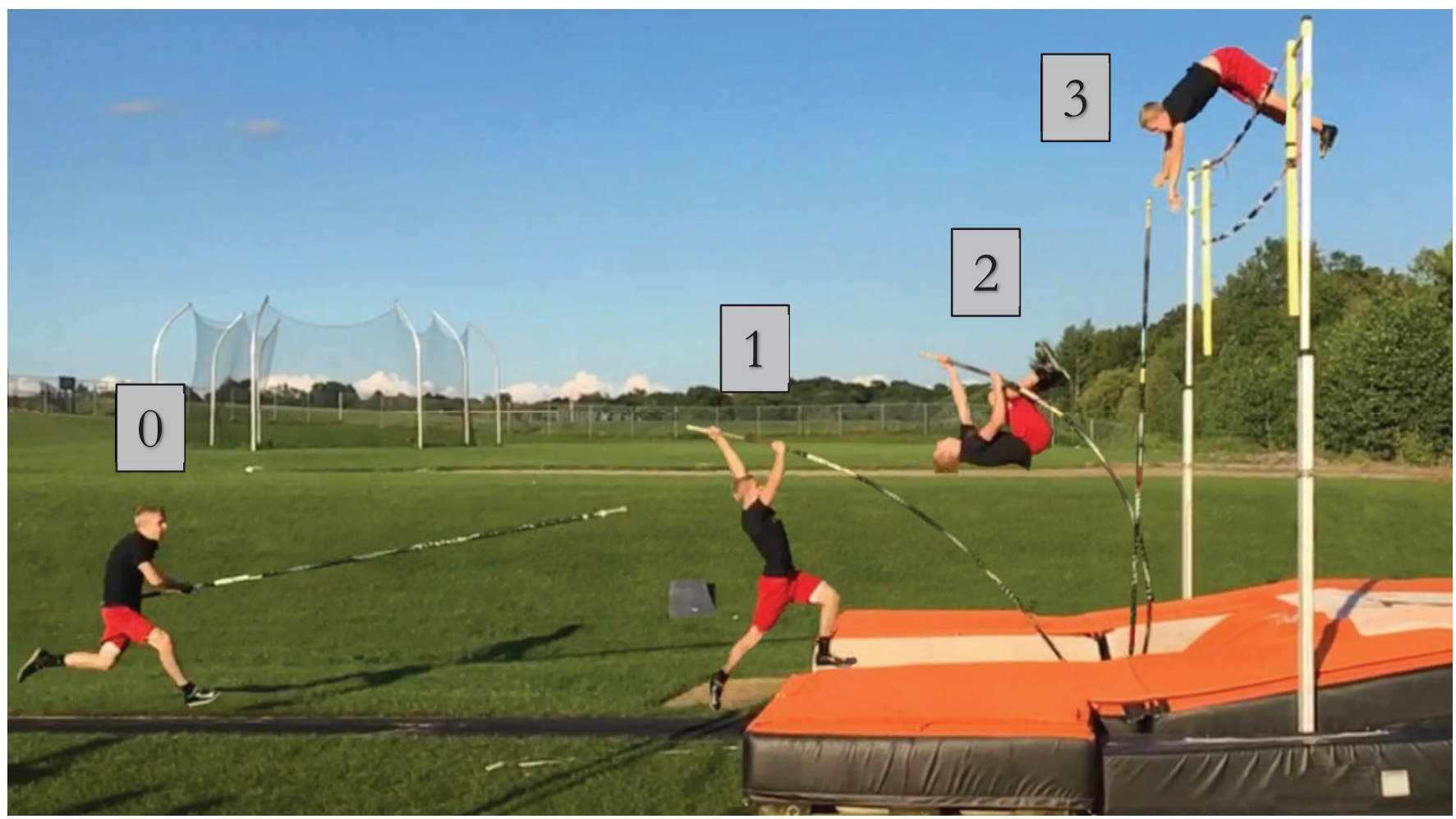

Figure 1. Pole vault stages and corresponding vault completeness scores. (Original photo)

The "SE1" measure was used because a vast majority of all first warmup jumps scored a one, suggesting participants were intending to score this as a form of a warmup drill. The "SL3" measure was used to test if the last three warmup vaults had more influence than the preceding ones. One point to note is that these measures included instances in which a participant did not make any height in a competition, commonly known as a "no height" (NH). Functionally, these scores represent the same as vaulting "zero" in competition. In an attempt to prevent these scores of zero skewing the correlative data, performance and scores were averaged for each participant then compared across participants.

An alternate analysis was then performed to address $\mathrm{NH}$ data in a different fashion that represented them as individual observation results without skewing the data set. This was done by ordering all of the performances for each athlete from highest to lowest (PerfRank) and comparing that order to the corresponding order of warmup scores (SRank), warmup scores except the first (SE1Rank), last three warmup scores (SL3Rank), and the total number of warmup vaults (TWUVRank) via Spearman's rank correlation.

\section{RESULTS}

Across 16 participants over the course of seven competitions, a total of 60 observations took place, with 15 resulting in a NH. The Pearson correlation between performance and TWUV (Figure 2) was significant, while no significant relationships were found between performance and Score, ScoreE1, or ScoreL3 (Table 2).

When comparing the data rank wise, significant Spearman's correlations were found between PerfRank and SRank, as well as PerfRank and SE1Rank, while no significant relationships were found between PerfRank and SL3Rank or TWUVRank (Table 2). 


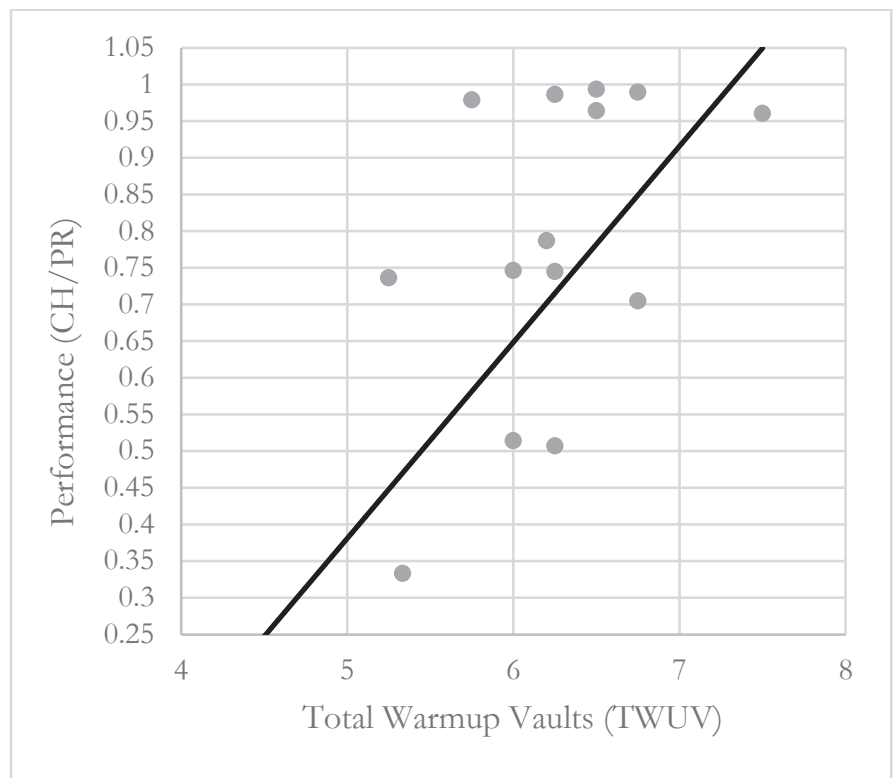

Figure 2. Scatterplot and trendline showing Pearson correlation between Total Warmup Vaults and Performance.

\begin{tabular}{|c|c|c|c|c|c|}
\hline \multirow{2}{*}{ Variable } & \multicolumn{2}{|c|}{ Performance } & \multirow{2}{*}{ Variable } & \multicolumn{2}{c|}{ Performance Rank } \\
\cline { 2 - 3 } \cline { 5 - 6 } & $r^{a}$ & $p$ & & $\varrho^{b}$ & $p$ \\
\hline S & .35 & .189 & SRank & .31 & $.014^{*}$ \\
\hline SE1 & .36 & .172 & SE1Rank & .32 & $.012^{*}$ \\
\hline SL3 & .43 & .099 & SL3Rank & .23 & .078 \\
\hline TWUV & .56 & $.029^{*}$ & TWUVRank & .19 & .148 \\
\hline
\end{tabular}

Table 2. Correlative results. $r$ - Pearson correlation coefficient, $\mathrm{p}$ - level of significance, $\varrho$ - Spearman's correlation coefficient, $S$ - average score of all warmup vaults, SE1 - average score of all warmup vaults except the first, SL3 - average score of the last three warmup vaults only, TWUV - total warmup vaults, Rank -

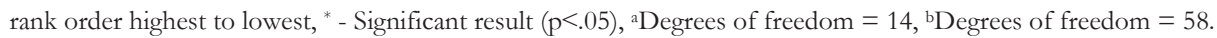

\section{DISCUSSION}

The results of this study support the hypothesis that better performance during warmups, as measured by vault completeness, is associated with better performance in competition. They also support the hypothesis that taking more total warmup vaults is associated with better competition performance.

Participants' best scores during the warmup period significantly correlated with their best competition performances. This was true when comparing the average of all the warmup jumps in a given competition as well as the average of all except the first warmup jump. When considering the number of warmup vaults taken before competition, participants who took more warmup jumps, on average, performed better in competitions over the course of the study. Had competition performance been a measure of the height vaulted, this result could easily have been attributed to confounding differences between participants. However, because performance was measured as a ratio comparing participants' performance to their own PR, this explanation is less likely.

One important feature to consider is the role NHs take within the interpretation of the results. NHs present a unique problem in that they are a qualitative representation of performance while all other marks are quantitative. This makes them challenging to draw meaningful comparisons between the associated data. To incorporate NHs as vaulting a height of "zero" would skew the data, while removing them from the data set altogether would lead to the consideration of only a "higher performance" subset of what actually occurs in competition. Either of these could explain why the Pearson correlations between performance and warmup scores failed to reach significance while the Spearman rank order correlations did. Including NHs in the average performance of each vaulter does create a useful means of comparison, but it also eliminates their significance as distinct observations which may lead to their influence being underrepresented. In light of this, there was still a significant Pearson 
correlation between performance and TWUV suggesting the effect was robust enough be present despite the data being averaged. In a similar manner, the Spearman's correlations between PerfRank and TWUVRank as well as SL3Rank were insignificant. This is likely due to the nature of the variables themselves. With TWUV ranging from 5-8, ranking led to a large number of ties. The same was true with SL3, where ranking the average of only three vaults led to a large number of ties.

It is worth noting that, while some of the relationships did reach significance, their correlation coefficients were not particularly high. The variables were compared using linear correlation, but it is entirely possible these relationships are more complex and require different analyses. Perhaps with longer observation periods constituting multiple pole vault seasons and a larger sample, the relationships will be better characterized for consideration of non-linear comparisons of the variables.

Low warmup vault scores suggest that a participant was unable to complete vaults due to inadequate performance of the preceding stages of the attempt. This is likely due to unfamiliarity with aspects of the competition environment relevant to proper execution of the earlier stages of the vault e.g. reaching a desired take-off target with unfamiliar cues. ${ }^{7,13}$ It may also be due to conditions external to the physical competition but still within the environment itself such as crowd size, temperature, time of year, stakes of the competition, skill of the competitors, etc. When considering the high level of experience participants have with the event, an environmental explanation becomes even more likely. This would suggest that those who had high warmup vault scores not only had higher environmental familiarity, but that this translated to better performance in competition, as the results support. In this context, it is worth noting that all of the data in the present study came from the same facility, an indoor fieldhouse which is the participants" most common place of competition (i.e., their "home" location). A valuable direction of future research would be to repeat this study at different competition venues to see if the effects are enhanced in alternate environments.

The main limitations of the study come from the challenge presented by analyzing NH data. To limit skewing the data, performances were averaged within participants at the expense of the representativeness of each individual observation. To combat this, performance was analyzed in terms of rank. This allowed the representation of NH data, but at the expense of analyzing relationships based on the magnitude of difference. A future direction of research could be collecting more data and using it to create a model by which NHs can represent a nonzero performance that can be meaningfully compared to others. Collecting more data may also help address the correlation coefficients of the significant findings and help to further characterize their relationship. To avoid adding any potential stress to the athletes, because data was collected in NCAA sanctioned competition, active involvement of participants in the research by way of surveys/questionnaires was not requested. Because of this, other factors such as weight, length, and flex of poles being used, documenting when each athlete switched poles, reflecting on warmups/competition performance, etc., were not considered. Future research observing athletes in unofficial competitions or practice would allow this limitation to be addressed.

\section{CONCLUSION}

To more effectively utilize warmup periods, coaches and athletes should consider adopting practices that focus on familiarizing oneself with their competition environment or simulating the competition environment during practice. Some examples of this may include: using the same runway and landing area that will be used in competition, recreating potential competition scenarios, taking more vaults from a full approach, using a competition bar in practice instead of a bungee, setting the standards to what an athlete normally uses in competition, and giving coaching instructions as one would in a competition. While these results do provide valuable insight into pole vault performance, it should be acknowledged that participants in the study constituted only a sample and attended the same university, experiencing similar training and coaching. When considering warmups, participants' best vault completeness scores coincided with their best competition performance scores. Also, participants who took more warmup jumps on average performed better in competition.

\section{ACKNOWLEDGEMENTS}

The author thanks Dr. Robert Deaner for his guidance and continued interest in the development of the author's research skills and knowledge throughout this project. The author also thanks Ellianne Martin, whose involvement was integral to the completion of this work.

\section{REFERENCES}

1. Lappin, J. S., Shelton, A. L., \& Rieser, J. J. (2006) Environmental context influences visually perceived distance. Perception \& Psychophysics, 68, 571-581. https:// doi.org/10.3758/BF03208759

2. Witt, J. K., Stefanucci, J. K., Riener, C. R., \& Proffitt, D. R. (2007) Seeing beyond the Target: Environmental context affects distance perception. Perception, 36(12), 1752-1768. bttps:// doi.org/10.1068/p5617 
3. Morone, G., \& Paolucci, S. (2012) Walking there: Environmental influence on walking-distance estimation. Behavioural Brain Research, 226(1), 124.

4. Epstein, W. (1978) Two factors in the perception of velocity at a distance. Perception \& Psychophysics, 24(2), 105-114. https:// doi.org/10.3758/BF03199536

5. Muffato, V., \& Meneghetti, C. (2020) Knowledge of familiar environments: Assessing modalities and individual visuo-spatial factors. Journal of Environmental Psychology, 67, 101387. https:/ / doi.org/ 10.1016/j.jemup.2020.101387

6. Pinder, R. A., Davids, K., Renshaw, I., \& Araújo, D. (2011) Manipulating informational constraints shapes movement reorganization in interceptive actions. Attention, Perception and Psychophysics, 73(4), 1242-1254.

7. Panteli, F., Smirniotou, A., \& Theodorou, A. (2016) Performance environment and nested task constraints influence long jump approach run: A preliminary study. Journal of Sports Sciences, 34(12), 1116-1123. bttps:/ / doi.org/ 10.1080/02640414.2015.1092567

8. Jamieson, J. P. (2010) The Home Field Advantage in Athletics: A Meta-Analysis. Journal of Applied Social Psychology, 40(7), 1819-1848. https:// doi.org/10.1111/j.1559-1816.2010.00641.x

9. Pollard, R. (2002) Evidence of a reduced home advantage when a team moves to a new stadium. Journal of Sports Sciences, 20(12), 969-973. bttps:// doi.org/10.1080/026404102321011724

10. Iosa, M., Fusco, A., Gudelj, I., Zagorac, N., \& Babić, V. (2013) Influence of Kinematic Parameters on Pole Vault Results in Top Juniors. Collegium Antropologicum, 37 Suppl 2, 25-30.

11. Johnson, H. W. (1961) Skill $=$ speed $\times$ accuracy $\times$ form $\times$ adaptability. Percept. Mot. Skills 13, 163-170.

12. Lee, T., Swanson, L., \& Hall, A. (1991) What Is Repeated in a Repetition? Effects of Practice Conditions on Motor Skill Acquisition, Physical Therapy, 71 (2) 150-156, https://doi.org/10.1093/ptj/71.2.150

13. Needham, L., Exell, T. A., Bezodis, I. N., \& Irwin, G. (2018) Patterns of locomotor regulation during the pole vault approach phase. Journal of Sports Sciences, 36(15), 1742-1748. https:/ / doi.org/ 10.1080/02640414.2017.1412236

\section{ABOUT THE AUTHOR}

Alex Peskin graduated from Grand Valley State University in April of 2020, dual majoring in Biomedical Science and Behavioral Neuroscience. He currently works as an oncology research coordinator and plans to pursue a medical degree.

\section{PRESS SUMMARY}

The pole vault is a track and field even where athletes use a long pole to launch themselves high in the air over a bar. In a pole vault competition, athletes have the opportunity to warm up in the same area and perform the same motions they will in the competition. The aim of this research was to determine if there was a link between an athlete's performance in warmups and in competition. After observing collegiate pole vaulters and tracking their performances, a link was found. Athletes who performed better in warmups also performed better in the competition. Also, athletes who took more practice runs performed better. These findings indicate that certain aspects of the warmup process could be important to an athlete's success in a competition. If athletes and coaches were to focus on key factors such as simulating the competition environment in practice and focusing on completing warmup vaults, they may have better performances. 